\title{
Beauty Sleep: Sleep Quality for Women is More than a Myth
}

\author{
Deborah A Goss* and Fariborz Ashtyani \\ Hackensack Sleep \& Pulmonary Center, USA
}

Submission: June 17, 2017; Published: July 13, 2017

*Corresponding author: Deborah A Goss, Hackensack Sleep \& Pulmonary Center, 170 Prospect Avenue, Suite 20, Hackensack, NJ, 07601, USA, Tel: 001112019960232; Fax: 001112019960095; Email:gossda0325@gmail.com

\section{Abstract}

The importance of sleep has always been recognized for daytime alertness and improved physical functioning. However, the effect of sleep on the body was frequently attributed to mainly affecting appearance, otherwise referred to as, "beauty sleep." It was not until the modern era that the essential need for sleep in order to have normal physiologic functioning, and the possible role of disrupted sleep in early morbidity and mortality were recognized.

It is not surprising, that this impact extends to hormonal regulation and function in the female body. It can affect everything from menarche to menopause, and even to the development of comorbidities in women.A brief and focused history and physical exam can alert the clinician to the presence of sleep disorders which can affect maternal-fetal health, worsen post-partum depression, and exacerbate insomnia postmenopausal women. Similarly, it is well known that sleep disorders can worsen diabetes, hypertension, and weight gain; which afflict women at all stages of life. With the advent of home sleep testing, it has become even easier to diagnose many sleep disorders. Importantly, many sleep disorders have effective treatments which not only result in the restoration of normal sleep physiology, but improve the restorative quality and beauty of sleep.

Keywords: Sleep; Fertility; Pregnancy; Menopause; Hormone; Sleep apnea; Insomnia

Abbreviations: AHI: Apnea Hypopnea Index; CPAP: Continuous Positive Airway Pressure; OSA: Obstructive Sleep Apnea; PLMS: Periodic Limb Movement Syndrome; RLS: Restless Legs Syndrome; SIDS: Sudden Infant Death Syndrome

\section{Introduction}

Sleep quality effects women's health during all stages of their lives, with a significant impact on reproductive years. From womb to tomb, sleep significantly affects the secretion and function of female hormones, which in turn, also affect the quality and quantity of sleep [1,2]. This relationship is not a static one. Constant and dynamic changes in body weight and proportion, oropharyngeal structure, hormone secretion, aging, inflammation, immunity and stress each have a role to play. While the importance of this relationship is being increasingly recognized, the physiologic pathways and neurobiological controls are incompletely understood and have not yet been fully elucidated.

In addition to biological factors, psychosocial and societal expectations and stereotypes regarding sleep also play a role. This significantly impacts on how women perceive their sleep and the presence of sleep disorders, as well as their approach to obtaining a diagnosis and treatment. The culmination of which can influence morbidity and mortality. Interestingly, sleep is both a voluntary and an involuntary physiologic process which can be enhanced or inhibited to a certain degree, at will [3]. In essence, to some extent, a woman can choose to become a "good" or "bad" sleeper by behavioral changes and medical interventions. The subsequent quality of a young woman's sleep may influence everything from school performance and neurocognitive functioning to weight gain, medical illness, depression, infertility, injuries, and even early death. The focus of this mini-review, is to highlight the basic physiology and pathophysiology of female sleep and the most current and cutting-edge treatment strategies. This will include: normal sleep in women, the effect of sleep on fertility, pregnancy, post-partum depression, insomnia, and the diagnosis and treatment of common sleep disorders.

\section{Discussion}

\section{Sleep in young women}

During infancy and early childhood, there are few significant differences in the timing, duration and composition of the sleep cycle that are noted in the medical literature. However, the 
striking prevalence of Sudden Infant Death Syndrome (SIDS) in male infants has led to further studies of gender difference in infant sleep. Interestingly, female infants are less likely to arouse from sleep when exposed to a brief jet of air from a nasal cannula. This difference resolves by age three months [4]. One theory for the cause of SIDS is that it results from an immature autonomic response to reflux, leading to prolonged apnea, cardiac dysrhythmia and death [5]. While GERD may be slightly more common in females, estrogen may have a protective, antiinflammatory effect against the damage it causes [6]. Similarly, bedwetting, sleep walking, and obstructive sleep apnea are also more common in boys, but only obstructive apnea has been directly linked to testosterone.

\section{Sleep and fertility}

Puberty is marked by a surge in sex hormones. However, the earliest changes which trigger puberty are thought to center around lipid-related hormones which are, in part, controlled by sleep. In girls, decreased leptin and increased body mass index are thought to lead to increases in growth hormone. The secretion of growth hormone is maximal during sleep [2,7]. There is also a significant increase in grey matter in the brain, especially in girls, which is loosely associated with NREM sleep [8]. Puberty may also lead to delayed bedtime, also known as circadian phase delay, which also decreases fertility.

Adult women have increased slow-wave sleep and increased sleep spindles, compared to men. There are differences in the timing of delta sleep [9]. This in combination with the above, may partially explain the propensity of women towards fibromyalgia, which is hallmarked by the presence of alpha-delta sleep. Another sleep disorder which is also affected by gender is obstructive sleep apnea. Men have an increased rate of sleep apnea, which is attributed to increased testosterone and its deleterious effect on upper airway muscular tone and patency. In comparison, women have a lower rate of sleep apnea, except in polycystic ovarian syndrome (PCOS) where the risk can increase by 30 - to 40 -fold. This is attributed to the high androgen levels that are seen in women with PCOS [10].

Sleep disruption (due to sleep apnea) and deprivation in adult women with PCOS (orin women who perform shift work) has been associated with increased dysmenorrhea, low fertility, increased miscarriages and lower birth rates. This is partly attributed to the disruption of normal reproductive hormones. Women with insomnia can also have decreased fertility. Ironically, melatonin used as a treatment for insomnia can also decrease fertility in these women, and should not be used to treat insomnia in women trying to attain pregnancy [10]. Diphenhydramine and zolpidem may be reasonable alternatives, but have not been studied for this purpose. Obstructive sleep apnea, due to PCOS, can be effectively treated with nocturnal continuous positive airway pressure or CPAP. While this can restore normal sleep, the extent to which it can improve conception rates has not been rigorously studied.
In summary, normal sleep is key in the achievement and maintenance of a healthy pregnancy. According to the current literature, it is suggested that all women desiring pregnancy try to maintain seven hours of non-disrupted sleep a night, and avoid late-night bedtimes and shift work. What is not yet clearly delineated in the literature is if women with sleep disorders can have their fertility restored by receiving treatment to restore normal sleep.

\section{Sleep and pregnancy}

High progesterone levels in the first trimester of pregnancy can increase daytime sleepiness and fatigue which is characteristic of early pregnancy [11]. As pregnancy progresses, there is significant weight gain and increased upper airway congestion and edema, which can lead to increased snoring and the development or worsening of obstructive sleep apnea. Gestational sleep apnea is linked to an increased risk for complications in pregnancy, including diabetes, hypertension and low birth weight. Whether this is a causal association is uncertain. There are also increased awakenings during pregnancy. This has been attributed to physical discomfort and to hormonal shifts in preparation for nocturnal feedings and infant care post-delivery.

While physical discomfort may disrupt sleep during any trimester, women who complain of discomfort should be asked particularly if they have increased discomfort in their lower extremities, as this can be a symptom of restless legs syndrome (RLS) periodic limb movement syndrome (PLMS). PLMS does occur at high rates in pregnancy, and is worse in the third trimester, when estradiol levels are maximally elevated [12]. However, it is also associated with iron deficiency anemia and uremia and screening labs for these disorders should also be performed. Treatment with iron and folate may improve symptoms in deficiency states, but frequently opioids are required for substantial relief with minimal data available on the best management and dosages.

Gastroesophageal reflux is also increased in sleep in pregnancy and may contribute to the increased incidence of asthma and pneumonia in this population. Diet and lifestyle changes may significantly improve reflux, with avoidance of late night eating and caffeine, in combination with elevation of the head of the bed. Calcium and magnesium supplements are frequently used for mild symptoms, escalating to H2-blockers and proton pump inhibitors for more severe symptoms [13].

\section{Sleep and post-partum period}

During young adulthood, many women will voluntarily restrict their sleep to pursue social activities. However, sleep disruption is significantly increased when caring for a newborn. When total sleep time is less than six hours a night, chronic sleep deprivation can occur. Over time, this can lead to impaired glucose metabolism, weight gain and hormonal imbalance [14]. With the addition of a sleep disorder, this is significantly 
magnified. It can lead to maternal and fetal complications [1517] and turn what should be a time of great happiness and joy, into a time of frustration, exhaustion and depression. For example, treatment of sleep apnea with CPAP may be impractical for a Mom who must leave her own bed very 2-3 hours to care for a newborn and then repeatedly replace her mask and restart her machine. Bed sharing (i.e., infant sleeping in same bed as Mom), is generally discouraged as this can lead to injury to the newborn and poor sleep for the parent.

In addition to medical sleep disorders, serious and potentially fatal psychological disorders, such as depression, bipolar disorder and psychosis, can be triggered and/or exacerbated by sleep loss [14]. These may be mitigated or exacerbated by lactation and breast feeding, which may lead to either improved satisfaction and mood, or frustration and increased sleep loss in which bottle-feeding may significantly improve maternal sleep. Post-partum depression has been found to be worsened by sleep loss, particularly with increased sleep latency and wake after sleep onset [18]. This is an interesting finding, as depression in general, is generally improved by restriction of total sleep time. More studies are clearly needed.

\section{Menopause and sleep}

The total quantity of slow wave sleep is diminished as women age. Slow wave sleep is restorative sleep, which results in feeling "refreshed" in the morning. Men tend to lose SWS at an earlier age than women do. After the age of 65 , about one third of women report increase difficulty falling asleep and increased awakenings during the night. Sleep disorders, including insomnia, obstructive sleep apnea, PLMS and circadian rhythm disorders also increased in this age group. The combination of the above resulting in difficulty falling asleep, frequent nocturnal awakenings, and then early morning awakening and late afternoon napping [11]. Treatment for sleep apnea in this age group is no longer limited to nocturnal mask ventilation. The availability of small implantable hypoglossal nerve stimulators now allows for a more comfortable and practical solution for women who are intolerant of nocturnal continuous positive airway pressure ventilation (CPAP).

The increase in obstructive sleep apnea may be directly related to the loss of estrogen, which promotes sleep and progesterone, which in addition to estrogen, also may be somewhat protective against apnea and stimulate breathing. It is the loss of estrogen which is also associated with the increased risk of insomnia. Oddly, the supplementation of estrogen and progesterone during menopause does little to improve sleep complaints [19], while targeted sleep therapies for insomnia and vasomotor symptoms during menopause are usually quite effective.

\section{Focused sleep history and physical exam}

As described above, there is a marked impact of sleep disorders on the health and well-being of women. It is therefore important for all clinicians caring for these patients to be able to effectively screen for these disorders and recognize when further diagnostic evaluation and treatment is warranted.

A basic sleep history includes and evaluation of the patient's bedroom environment, sleep hygiene, timing of sleep onset, sleep duration, sleep disruption and wake times. It is recommended that the sleep environment be a cool $\left(65^{\circ} \mathrm{F}\right.$ or $18{ }^{\circ} \mathrm{C}$ ) room which is quiet and dark [20]. Light, including both sunlight and electronics, is to be avoided. Sleep onset should be within 30-60 minutes of entering the bed and sleep duration is recommended to be approximately seven hours. If there is a prolonged awakening, the sleeper is encouraged to leave the bedroom and engage in a quiet and non-stimulating activity and return to bed when sleepy. The amount and frequency of sleep disruption during the night should also be noted.

On physical exam, pupil size inconsistent with the luminosity of the room may be related to either excessive daytime sleepiness or to substance abuse. It is not uncommon for patients with sleep disorders to self-medicate with diphenhydramine, melatonin, caffeine, alcohol or other substances. It is worth inquiring with patients what non-prescriptive and/or herbal treatments they are using. The external neck circumference [21] as well as the narrowness of the oropharyngeal airway is also associated with sleep apnea [22]. While, morbid obesity increases the likelihood of sleep apnea, but even patients of normal weights can have severe apnea. However, for the non-Sleep clinician, the use of the STOP-BANG survey and Epworth Sleepiness Scale may be the easiest and effective basic screenings for sleep disorders [23].

\section{Conclusion}

It has been clearly shown that the quality and quantity of sleep can have a dramatic impact on women's health. The treatment of an underlying sleep disorder, can improve a patient's daytime functioning, and lead to improvement in the management of obesity, depression, diabetes and hypertension. Sleep disorders can be easily screened for by clinicians with a brief clinical assessment and effectively treated. It is also clear that having an adequate quantity of quality sleep can lead to improved physical and mental health, as well as decreased weight, and even improved appearance (with decreased skin aging and improved recovery from UV light exposure [24]. It therefore appears that the ugly truth is that quality sleep is essential for the normal function of the female body, and revealing the beauty without and within.

\section{Acknowledgements}

Special thanks to Dr. Abdulla Al-Khan from the Department of Maternal Fetal Medicine at Hackensack University Health Center for many informative conferences and multidisciplinary management of women with complex pulmonary and sleep disorders in pregnancy. 


\section{Conflict of Interest}

Dr. Goss- None. Dr. Ashtyani- None.

\section{References}

1. de Zambotti M, Colrain IM, Baker F (2015) Interaction between reproductive hormones and physiological sleep in women. J Clin Endocrinol Metab 100(4): 1426-1433.

2. Finlayson CA, Styne DM, Jameson JL (2016) Endocrinology of sexual maturation and puberty. Elsevier. Wilton, Connecticut, pp. 2119 2129 e2.

3. Copinschi G, Challet E (2016) Endocrine rhythms, the sleep-wake cycle, and biological clocks. Elsevier Wilton, Connecticut 147-173e9.

4. Richardson HL, Walker AM, Horne RS (2010) Sleeping like a baby- does gender influence infant arousability? Sleep 33(8): 1055-1060.

5. Jolley SG, Halpern LM, Tunell WP, Johnson DG, Sterling CE (1991) The risk of sudden infant death from gastroesophageal reflux. J Pediatr Surg 26(6): 691-696.

6. Kim YS, Kim N, Kim GA (2016) Sex and gender differences in gastroesophageal reflux disease. J Neurogastroenterol Motil 22(4): 575-588.

7. Garcia-Garcia F, Juarez-Aguilar E, Santiago-Garcia JS, Cardinali DP (2014) Ghrelin and its interactions with growth hormone, leptin, and orexins: implications for the sleep-wake cycle and metabolism. Sleep Medicine Reviewes 18(1): 89-97.

8. Feinberg I, Campbell IG (2010) Sleep EEG changes during adolescence: an index of a fundamental brain reorganization. Brain Cogn 72(1): 5665.

9. Andrea D (2014) Women's Health. In: Kasper D, Fauci A, Hauser S, Longo D, Jameson J, et al. (EDs.), Harrison's Principles of Internal Medicine. McGraw-Hill, New York, USA, p. 19.

10. Kloss JD, Perlis, M, Zamzow J, Culnan E, Gracia C (2016) Sleep, sleep disturbance and fertility in women. Sleep Med Rev 22: 78-87.

11. Clark CP, Moore PJ, Gillin J (2008) Sleep disorders. In: Ebert MH, Loosen PT, Nurcombe B, Leckman JF (Eds.), Current diagnosis \& treatment: Psychiatry. McGraw-Hill, New York, USA, p. 336.

12. Wehrle R, Lancel M, Pollmacher T (2009) Elevated estradiol plasma levels in women with restless legs during pregnancy. Sleep 32(2): 169174 .

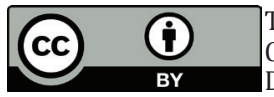

This work is licensed under Creative

Commons Attribution 4.0 Licens

DOI: 10.19080/JGWH.2017.06.555676
13. Richter JE (2005) Review article: the management of heartburn in pregnancy. Aliment Pharmacol Ther 22(9): 749-757.

14. Jameson JL, De Groot LJ (2015) Conditions of altered sleep and circadian rhythmicity sleep curtailment. In: Endocrinology: Adult and Pediatric E-Book. Elsevier, New York, USA, p. 170.

15. Cain MA, Louis JM (2016) Sleep disordered breathing and adverse pregnancy outcomes. Clin Lab Med 36(2): 435-446.

16. Louis JM, Auckley D, Sokol RJ (2010) Maternal and neonatal morbidities associated with obstructive sleep apnea complicating pregnancy. Am ] Obstet Gynecol 202(3): 261.

17. Hutter D, Davis E, Alvarez M, Ashtyani H (2004) Use of noninvasive positive pressure during wakefulness for the treatment of hypertension in pregnancy. Chest $126(4)$.

18. Posmontier B (2008) Sleep qualityin women with and without postpartum depression. JOGNN 37(6): 722-737.

19. Kalleinen N, Polo O, Himanen SL, Joutsen A, Polo-Kantola P (2008) The effect of estrogen plus progestin treatment on sleep: a randomized, placebo-controlled, double-blind trial in premenopausal and late postmenopausal women. Climateric 11(3): 233-243.

20. Lack LC, Gradisar M, Van Someren EJ, Wright HR, Lushington K (2008) The relationship between insomnia and body temperature. Sleep Med Rev 12(4): 307-317.

21. Davies RJ, Stradling JR (1990) The relationship between neck circumference, radiographic pharyngeal anatomy, and the obstructive sleep apnoea syndrome. Eur Respir J 3(5): 509-514.

22. Nuckton TJ, Glidden DV, Browner WS, Claman DM (2006) Physical examination: Mallampati score as an independent predictor of obstructive sleep apnea. Sleep 29(7): 903-908.

23. Chiu HY, Chen PY, Chuang LP, Chen NH, Tu YK, et al. (2016) Diagnostic accuracy of the Berlin questionnaire, STOP-BANG, STOP and Epworth sleepiness scale in detecting obstructive sleep apnea: a bivariate metaanalysis. Sleep Med Rev 16(30): 3065-3070.

24. Baron E (2013) Effects of sleep quality on skin aging and function. In: Proceedings of the International Investigative Dermatology Meeting Sponsored by Estee Lauder. University Hospitals (UH) Case Medical Center, Edinburgh, Scotland, UK.

\section{Your next submission with Juniper Publishers} will reach you the below assets

- Quality Editorial service

- Swift Peer Review

- Reprints availability

- E-prints Service

- Manuscript Podcast for convenient understanding

- Global attainment for your research

- Manuscript accessibility in different formats

( Pdf, E-pub, Full Text, Audio)

- Unceasing customer service

Track the below URL for one-step submission https://juniperpublishers.com/online-submission.php 